\title{
Ethnologies
}

\section{L'adaptation des soins sociosanitaires de première ligne à la diversité religieuse}

\section{L'exemple du Québec}

\section{Josiane Le Gall et Spyridoula Xenocostas}

Volume 33, numéro 1, 2011

URI : https://id.erudit.org/iderudit/1007801ar

DOI : https://doi.org/10.7202/1007801ar

Aller au sommaire du numéro

Éditeur(s)

Association Canadienne d'Ethnologie et de Folklore

ISSN

1481-5974 (imprimé)

1708-0401 (numérique)

Découvrir la revue

Citer cet article

Le Gall, J. \& Xenocostas, S. (2011). L’adaptation des soins sociosanitaires de première ligne à la diversité religieuse : l'exemple du Québec. Ethnologies, 33(1), 169-189. https://doi.org/10.7202/1007801ar
Résumé de l'article

La diversité religieuse croissante de la population québécoise soulève certaines questions essentielles pour les institutions médicales, dont celle de leur adaptation aux différences religieuses. À partir de cas cliniques recueillis lors d'une recherche récente sur les défis et enjeux posés par la diversité religieuse dans l'intervention de première ligne, nous examinons dans cet article la manière dont la diversité religieuse est prise en compte par les intervenants dans leur travail. Nous cherchons également à voir comment se manifeste la religion dans la rencontre clinique et quelles sont les réactions des intervenants face aux requêtes des usagers. 


\section{L'ADAPTATION DES SOINS SOCIOSANITAIRES DE PREMIÈRE LIGNE À LA DIVERSITÉ RELIGIEUSE \\ L'exemple du Québec}

Josiane Le Gall

Département de communication publique et sociale, UQAM

Spyridoula Xenocostas

Directrice du Centre de recherche et de formation (CRF), CSSS de la Montagne

Dans de nombreuses sociétés contemporaines, le paysage religieux a récemment connu de profondes modifications, notamment sous l'impact des migrations. La présence de groupes religieux minoritaires soulève de nouvelles interrogations et interpelle les institutions publiques. Par exemple, pour les institutions sociosanitaires, l'adaptation des soins aux nouvelles caractéristiques religieuses de la clientèle pose un nouveau défi (Oxman-Martinez et Hanley 2005). La diversification religieuse de la population se manifeste par de multiples conceptions religieuses de la santé, de la maladie, de la souffrance, de la guérison et des besoins et attentes de santé spécifiques. Les liens multiples entre religion, santé et bien-être, de même que l'importance de la religion pour plusieurs natifs et immigrants, laissent entrevoir la possibilité que le religieux traverse la rencontre clinique. Néanmoins, les recherches en santé qui intègrent la dimension religieuse examinent surtout l'impact de celle-ci et soulignent généralement le lien favorable entre la pratique religieuse et le bien-être des personnes (voir Chatters 2000 ; Cochrane 2006). Très peu analysent l'incidence du pluralisme religieux sur l'organisation et la prestation des services de santé (Battaglini 2005; Pesut et Reimer-Kikham 2010). À partir d'une recherche menée à Montréal auprès d'intervenants œuvrant dans des Centres de santé et de services sociaux $(\mathrm{CSSS})^{1}$, cet article vise à examiner l'adaptation

1. Les Centres de santé et de services sociaux (CSSS) ont pour mandat d'offrir, à proximité du milieu de vie de la population dont ils ont la responsabilité, une 
des soins sociosanitaires de première ligne à la diversité religieuse. Après un survol de la littérature pertinente sur la question et une description de l'approche méthodologique adoptée, notre contribution portera d'abord sur la prise en compte par les intervenants de la diversité religieuse dans leur travail, pour ensuite aborder leurs réponses aux requêtes des usagers.

\section{Questionnement chez les professionnels de la santé}

À l'instar de quelques auteurs (voir Hall 1997 ; Orsi 2003), nous considérons qu'il est important d'étudier ce que Cadge et Ecklund (2007) nomment la « religion vécue », soit de saisir comment les individus vivent leur religion au quotidien en dehors des lieux de culte, notamment dans les entreprises, le quartier, les organisations politiques ou l'école. Le rapport des personnes croyantes aux institutions sociosanitaires a peu été étudié, et ce, tant du point de vue des usagers que de celui des professionnels. De rares recherches ont souligné comment la religion peut modifier la décision d'accepter ou non des soins de santé ou encore les modes d'utilisation et l'accessibilité des services, tant chez les populations natives que chez les immigrants (Barnes et Sered 2005 ; Curlin et al. 2005 ; Fadiman 1998 ; Koenig et al. 2001 ; Ong 1995).

L'impact de la religion sur le soin aux patients, tout comme la façon dont les professionnels de la santé conçoivent la religion, font également l'objet de peu d'études (Cadge et al. 2009). On ignore en effet comment ces derniers s'ajustent (ou pas) aux pratiques et croyances religieuses des clientèles. Quelles sont leurs réponses à la diversité religieuse ? Où trouvent-ils l'information sur la religion et comment déterminent-ils si elle est importante pour leur travail ? Quelles stratégies ou solutions proposent-ils ou adoptent-ils? Perçoivent-ils la religion comme une barrière ou comme un levier? Les différences dans les conceptions de la santé, de la maladie, de l'éthique qui peuvent exister entre les personnes croyantes et certains professionnels s'avèrent parfois sources de difficultés dans la rencontre clinique (Buryska 2001 ; Cochrane 2006 ; Pesut et Reimer-Kirkham 2010). En fait, il ne semble pas exister

large gamme de services de première ligne avec des mécanismes de référence et de suivi pour les services de $2^{\mathrm{e}}$ et de $3^{\mathrm{e}}$ lignes, soit les services spécialisés et surspécialisés. 
de consensus sur la place que la religion doit occuper dans les soins et celle-ci donne lieu à un questionnement chez les professionnels, qui peuvent avoir leurs propres croyances par ailleurs (Pesut et ReimerKirkham 2010). Dans une récente étude américaine sur la place de la religion et de la spiritualité dans le travail des pédiatres, Cadge et al. (2009) montrent comment ces derniers jugent que ces dimensions, malgré leur pertinence, se situent en dehors de leurs compétences professionnelles. Selon les circonstances, les frontières entre la religion et leur travail deviennent plus ou moins étanches : la religion appuie l'intervention dans des situations de fin de vie, mais devient un obstacle lorsqu'elle entrave un traitement médical. L'intervention sociosanitaire s'inscrit dans un cadre institutionnel où se définissent normes, règles, objectifs et orientations de niveau institutionnel, ainsi que dans un cadre plus large, sociopolitique. L'espace clinique est non seulement un espace de soins, mais aussi un espace social, relationnel, où normes, valeurs, modèles professionnels, sociaux et culturels se rencontrent (Fortin 2008). Cette perspective questionne la neutralité de l'espace clinique et rejette les explications culturalistes ou essentialistes pour comprendre les enjeux cliniques, tout en insistant sur la religion et l'ensemble des dimensions qui se juxtaposent à celle-ci dans l'intervention (par exemple pays d'origine, parcours migratoire, culture, classe sociale, genre, niveau d'éducation, degré de familiarité avec les normes bureaucratiques, organisationnelles et déontologiques du système de santé, rôles et mandat du professionnel). Il est donc essentiel de saisir non seulement le sens donné par les intervenants à la religion dans leur expérience, mais aussi les enjeux contextuels qui sous-tendent la rencontre clinique, tant sur le plan institutionnel que sociétal.

\section{Diversité religieuse au Québec et système de santé}

L'adaptation des soins et services fait partie de la gestion quotidienne des activités des établissements de santé et de services sociaux du Québec, en particulier à Montréal, où le paysage religieux connaît un processus de changement profond à la suite de la diversification des pays sources d'immigration (Mossière et Meintel 2010). Comme le rappellent Mossière et Meintel (2010), les quelques travaux empiriques sur les pratiques, croyances et rituels de groupes religieux implantés au Québec depuis les années 1960 soulignent la pertinence de la religion comme symbolique significative, mobilisatrice de liens sociaux et comme dimension identitaire pour de nombreux natifs et migrants, ce 
que nous avons eu l'occasion de constater dans nos recherches (Fortin, LeBlanc et Le Gall 2008 ; Le Gall 2003). Afin de compléter ce portrait, il est utile de rappeler les débats récents au Québec sur la place de la religion dans l'espace public, débats suscités en partie par l'affirmation de l'identité religieuse dans certaines sphères de la société.

Parce qu'ils adhèrent aux principes énoncés dans les Chartes des droits et libertés qui régissent le Québec, les établissements de santé doivent respecter les droits garantis par celles-ci et prodiguer des soins et services accessibles et appropriés à tous, dans une perspective d'équité et de justice sociale. De même, l'adaptation des services de santé à la diversité religieuse fait partie intégrante de la législation québécoise. Les établissements ont pour fonction "d'assurer la prestation de services de santé ou de services sociaux de qualité, qui soient continus, accessibles, sécuritaires et respectueux des droits des personnes et de leurs besoins spirituels et qui visent à réduire ou à solutionner les problèmes de santé et de bien-être et à satisfaire les besoins des groupes de la population» (LSSS 2006). Les intervenants semblent bien s'acquitter de l'obligation des établissements de respecter la liberté d'expression religieuse de leur clientèle alors que très peu de litiges se judiciarisent (Azdouz 2007). Dans le contexte de la prestation de soins et services de santé aux usagers, les intervenants sont amenés au quotidien à faire des adaptations qui tiennent compte de la condition clinique de la personne, de son histoire de vie, de ses besoins et de ses croyances. Il est donc de leur responsabilité de personnaliser les soins et services en respectant le caractère unique de l'individu et en lui offrant des soins adaptés. Pour adapter leurs pratiques, les professionnels de la santé doivent prendre plusieurs éléments en compte, dont notamment les demandes du client, leurs mandats professionnels et celles de leurs équipes, les orientations de l'établissement ou encore les politiques publiques. Par exemple, la loi limite l'adaptation des services aux possibilités des ressources humaines, matérielles et financières dont disposent les établissements de santé pour répondre à la demande. En raison de la grande diversité des croyances et pratiques religieuses de la clientèle, il n'existe donc pas une seule façon d'adapter les pratiques à la diversité religieuse, d'où l'intérêt d'examiner la prise en compte (ou non) de la dimension religieuse dans le travail des intervenants ainsi que les pratiques développées par rapport aux valeurs religieuses des usagers. 
Des intervenants de première ligne

Les données utilisées ici ont été recueillies lors d'un projet de recherche ${ }^{2}$ développé à la suite de journées de réflexion sur l'adaptation de l'intervention à la diversité culturelle organisées par le CSSS de la Montagne à Montréal en 2008 dans la foulée de la controverse sur les accommodements raisonnables. Lors de cet événement, la majorité des cas cliniques problématiques signalés par les intervenants touchaient les croyances ou pratiques religieuses des usagers. La recherche, portant sur les défis et enjeux posés par la diversité religieuse dans l'intervention de première ligne, cherchait à dégager les manifestations de la religion, les différentes questions soulevées par ces situations et les stratégies et solutions proposées ou adoptées par les professionnels. Trente-huit entretiens semi-dirigés ont été réalisés auprès d'intervenants œuvrant dans six CSSS montréalais ayant sur leur territoire, bien qu'à des degrés divers, une clientèle pluriethnique. Les profils rencontrés sont variés. Les répondants proviennent de différentes disciplines (infirmières, nutritionnistes, psycho-éducateurs, psychologues, travailleurs sociaux, sages-femmes, éducateurs, auxiliaires familiaux, organisateurs communautaires) et évoluent dans divers programmes et secteurs d'intervention (enfance-famille, soutien à domicile, DI-TED ${ }^{3}$, scolaire, services généraux adultes, maison des naissances, etc.). La plupart possèdent plusieurs années d'expérience, parfois dans d'autres secteurs d'intervention. Tous les âges sont représentés, bien que la génération des plus de 45 ans prédomine nettement. La majorité des répondants sont des femmes $(30 / 38)$ et près des deux tiers appartiennent au groupe majoritaire (franco-québécois) (25/38), tandis que les autres sont immigrants. Une grande diversité est également observée au niveau de leur religiosité : selon les cas, ils se déclarent sans religion, croyants et pratiquants ou croyants (principalement de confession chrétienne). Pour terminer ce portrait, précisons qu'une des stratégies de recrutement consistait à demander aux professionnels de soumettre des cas d'intervention soulevant des défis inhérents à l'adaptation des pratiques envers une clientèle diversifiée sur le plan religieux. De plus, tous ont

2. Le projet, intitulé «Diversité religieuse et intervention de première ligne »a bénéficié du financement du CRSH (RDI 2009). L'équipe de chercheurs est composée de Josiane Le Gall, Jacques Rhéaume, Vania Jimenez et Spyridoula Xenocostas. Les entretiens ont été réalisés par Sophie Lemoyne-Dessaint et Amélie Normandin.

3. Déficience intellectuelle et trouble envahissant du développement. 
été recrutés sur une base volontaire, ce qui peut témoigner chez eux d'une certaine ouverture à la religion et constituer par le fait même une des limites de la recherche.

\section{"Ce n'est pas moi qui décide de la place de la religion »}

Bien que le niveau de sensibilité des intervenants rencontrés lors de la recherche par rapport au fait religieux varie fortement, tous s'estiment plutôt ouverts et relativement à l'aise d'en parler dans le cadre de leur travail. Ils reconnaissent également les conséquences positives de la religion pour les individus, même si, selon certains, elle s'accompagne parfois d'une certaine « rigidité ». Ils mentionnent y avoir fait appel à un moment ou à un autre de leur pratique. Néanmoins, très peu traitent spontanément de la question ou l'explorent en détail avec leurs patients. Par exemple, Myriam, une psychologue employée aux services généraux adultes, s'informe parfois des croyances et pratiques religieuses des usagers, mais précise : "Je ne le fais pas de façon systématique, parfois je me dis que je devrais le faire ». Plusieurs intervenants devant remplir le formulaire d'évaluation de l'autonomie multiclientèle lors d'une demande de service omettent la section sur les croyances et valeurs spirituelles (et les aspects qui posent problème) ou ne font que l'effleurer. La plupart admettent d'ailleurs accorder peu de place à la religion, comme Frédéric, un psycho-éducateur en enfancefamille: "Il faut que ça se présente dans un contexte x. Puis on est pas très intrusif, à savoir, "parlez-nous de votre religion" ?»

La majorité des répondants qui abordent la question des croyances et des pratiques religieuses de leurs clients le font de façon indirecte, c'est-à-dire si ces derniers l'introduisent dans la discussion ou, parfois, si leurs croyances apparaissent comme un enjeu. Sonia, une travailleuse sociale en milieu scolaire, s'exprime à ce propos : "Ce n'est pas ma porte d'entrée. Ma porte d'entrée est de m'expliquer ce dans quoi vous êtes, comment puis-je comprendre pour que je vous aide». Comme plusieurs, elle n'intègre cette dimension que «si c'est là, mais ce n'est pas moi qui vais aller le chercher. [...] Ce n'est pas moi qui définis cette place-là. Je laisse la personne se situer ». Miranda, une travailleuse sociale née à l'étranger travaillant auprès de personnes âgées en perte d'autonomie et en perte cognitive dans un secteur de la ville qui a vu sa population se transformer récemment avec l'arrivée d'un nombre croissant de musulmans, partage un avis similaire. Elle se dit athée, 
mais ouverte et à l'aise de parler de religion avec les usagers, mais ne l'utilise que si elle joue un rôle primordial dans leur vie: «Non, je ne demande jamais l'appartenance [religieuse], mais si je vois que c'est présent et que ça a une place importante pour la cliente ou le client, là je l'aborde ». La présence de la religion dans l'intervention dépend donc en grande partie de la place que lui donnent les clients, comme en témoigne à nouveau Frédéric: "Ce n'est pas moi qui décide de la place de la religion. Je laisse toujours la cliente voir combien c'est important [dans sa vie]».

Seuls quelques professionnels abordent directement le fait religieux et interrogent parfois leurs clients sur le sujet. Toutefois, avant d'en discuter avec eux, ils s'assurent d'abord de leur religiosité, en se basant notamment sur la présence de signes religieux. Selon Myriam, certains indices, comme la tenue vestimentaire, en particulier chez les musulmans ou les juifs orthodoxes, témoignent de l'importance de la religion dans la vie des individus. Quelques clients effleurent également le sujet. «Parfois, si je leur parle de leurs habitudes de vie, il y en a qui vont dire, par exemple, qu'elles font de la méditation [...] parfois, ils vont le mentionner ouvertement: "dimanche je suis allée à la messe puis telle chose s'est passée” ». En l'absence de tout indice, seuls de très rares répondants explorent la question. Interrogée sur la place occupée par la religion dans la vie de ses clients, Nancy, une ergothérapeute au soutien à domicile, répond qu'il ne faut rien prendre pour acquis : «Je pense que c'est... au cas par cas, on va vérifier si c'est important pour eux, qu'est-ce que ça signifie? Qu'est-ce que ça veut dire? Quelle couleur ça peut prendre vraiment?». Toutefois, pour ces professionnels, la règle absolue consiste à attendre qu'un lien de confiance soit établi, ce qui nécessite souvent plusieurs rencontres.

\section{"Pas assez crédible pour parler de religion "}

Si la dimension religieuse n'apparaît pas dans l'intervention, et nous verrons plus loin qu'il s'agit d'une situation fréquente, la plupart des répondants ne chercheront donc pas à connaître l'importance ou le sens qu'elle revêt dans la vie des usagers. D'ailleurs, plusieurs avouent souvent ignorer leur appartenance religieuse. Pour ces personnes, la religion en tant que telle ne relève pas de leur mandat professionnel et ne tient pas d'emblée une place prépondérante dans la résolution de problèmes. Elles mentionnent d'ailleurs leur inconfort à l'introduire dans 
l'intervention. Par exemple, Frédéric explique qu'il aurait l'impression de manipuler le client et emploie à quelques reprises les termes «convaincre » et « convertir » pour étayer son propos. De plus, il juge trop faibles ses connaissances sur le sujet pour paraître crédible: «Je ne suis ni un pasteur, ni un prêtre, ni rien de tout ça et je n'ai pas assez de connaissances de toute façon pour aller dans ce panier-là ». Même les intervenants qui interrogent directement leurs clients sur leurs appartenances religieuses semblent éprouver un certain malaise à le faire, d'où le besoin d'attendre qu'un lien se crée ou de vérifier si la personne devant soi est pratiquante. Marie, catholique, qui a régulièrement recours à la religion dans son intervention, exprime à plusieurs reprises dans l'entrevue le sentiment d'imposture qu'elle ressent quant à son intérêt pour les croyances des clients, comme si elle devait justifier pourquoi elle s'y intéresse et la légitimité de ses questions. De même, Ariane, une travailleuse sociale du programme DI-TED qui se déclare athée, témoigne de son inconfort à utiliser la religion dans le cadre de ses fonctions : "Comme on est employé par un organisme qui est laïque, on ne peut pas aller loin dans ça ». Lévaluation psychosociale lui permet d'introduire la question, sans déborder des normes organisationnelles : «Un des items est, quel est le rôle de la spiritualité ? Ou avez-vous un réseau de support? Je pose la question avec plus de confiance parce que c'est là, c'est donné par le CLSC ».

Les personnes interviewées évoquent parfois la réaction du client pour justifier pourquoi, dans leur travail, elles ne recourent pas de façon systématique à la dimension religieuse. Dans un contexte où la religion suscite de vifs débats publics, certains professionnels hésitent à en parler de peur de rompre le lien thérapeutique. Ils veulent éviter de paraître trop indiscrets ou de heurter les usagers, qui pourraient se sentir confinés dans des catégories ou avoir l'impression qu'on remet leur relation à Dieu en question. Par exemple, quelques répondants déclarent que les usagers se sentiraient jugés s'ils investissaient trop tôt cette dimension dans la relation d'aide. Mélanie, une jeune travailleuse sociale d'enfancefamille, tient les discours médiatiques pour responsables de cette attitude.

Moi j'y vais un petit peu avec l'instinct, quand je sens qu'il y a un climat de confiance, ils savent aussi combien... on n'a pas les mêmes croyances religieuses, fait que je pense qu'avec leurs bagages aussi, je veux dire, ils lisent les journaux, ils ont vu tout ce qui se passe aussi par rapport aux accommodements raisonnables, tout ça. Ils ne savent 
peut-être pas comment nous on se situe. Une Blanche qui arrive avec ses croyances et qui travaille pour le gouvernement.

Parfois, des réticences surgissent du côté des clients. Ariane revient sur le cas d'une dame hassidique qui, en raison possiblement de sa conception du service d'aide, la trouvait intrusive : "Elle n'acceptait pas que je pose des questions sur la religion, sur les traditions, pour la connaître plus. Pour elle, pourquoi vouloir les connaître ? Pourquoi ? Quel intérêt j'ai à les connaître?»

\section{Un levier permettant de rejoindre et d'aider l'autre}

Interrogés sur les motifs qui les amènent à intégrer la religion dans leur travail, les répondants précisent que pour répondre aux besoins des usagers, ils doivent tenir compte de leurs préoccupations, que ce soit la religion ou un autre aspect. Lorsque ceux-ci en parlent, elle devient donc pertinente et représente une voie vers la résolution du problème rencontré. Lors de l'évaluation initiale, si Louise, une travailleuse sociale au soutien à domicile, réalise que la religion représente une force pour le client ou si ce dernier exprime des attentes à ce niveau, elle "ouvre » sur le sujet, une expression maintes fois employée. Comme d'autres, elle considère que son code d'éthique exige le respect des croyances, religieuses et autres, de ses clients : "On intervient à domicile dans leur milieu, avec leurs valeurs, leur histoire de vie, puis on sait qu'elle n'a pas commencé à notre arrivée ». Quelques-uns de ces professionnels, dont le niveau de connaissances sur les différents groupes religieux présents à Montréal varie, interrogent leurs clients lorsqu'ils soupçonnent que leurs croyances et pratiques religieuses représentent un enjeu dans la situation, en particulier un frein «à s'engager dans un processus ». Ils cherchent à mieux comprendre afin d'être en mesure d'apporter un soutien à l'autre. Comment perçoit-il la maladie ou le handicap ? Quel sens donne-t-il à la perte d'un proche? Il semble donc plus facile d'aborder la religion lorsqu'elle se manifeste de façon concrète dans l'intervention. Cela devient alors une donnée utile et nécessaire à considérer. Par exemple, dans le cadre de son travail de nutritionniste, Katia doit régulièrement interroger ses clients sur les liens entre leurs habitudes alimentaires et la religion pour bien saisir la complexité de la situation.

Seuls quelques répondants, en particulier les personnes qui abordent directement la religion avec les clients, vont aussi essayer de tirer profit 
du réconfort qu'elle peut apporter aux individus. Elle devient alors un levier pour rejoindre l'autre et créer une complicité, comme en témoigne Frédéric qui donne l'exemple d'une cliente algérienne avec laquelle il a ainsi réussi à créer un lien : "On dirait que ça défait des barrières de cette façon-là. [...] Des fois je suis bien au courant, mais je fais le naîf pour que les gens m'en parlent aussi ». La religion peut aussi être utilisée pour aider l'autre à surmonter un problème et cheminer. Depuis peu, Éloïse met de l'avant la religion, car même si elle se dit athée, elle croit que la foi a le pouvoir de soutenir et de réconforter : «Je me suis essayée, puis essayée, puis essayée. Puis là, de plus en plus, je pose la question [...] je crois que la foi peut les aider, que la religion peut les aider». Elle constate les gains obtenus en procédant de la sorte auprès des personnes croyantes: «Et ça va bien quand même, ça ouvre une autre porte. Ça ouvre à une certaine richesse en intervention que je n'avais pas. Disons au niveau du lien ça connecte aussi, plus à un certain niveau, où ils ont plus l'impression d'être compris et pas jugés ». Quant à Simon, un travailleur social intervenant auprès de réfugiés et qui dit avoir " fini de croire en Dieu depuis longtemps ", il met de l'avant le rôle positif joué par la religion dans la vie de ses clients, qu'il considère comme un levier thérapeutique.

Je trouve que c'est extrêmement positif parce que c'est toute la matrice qui leur donne espoir, qui leur donne du sens dans ce qui, a priori, n'a pas de sens. La religion, pour eux, c'est comment dire, l'épaule sur laquelle ils peuvent se reposer le temps de trouver du sens à ce qui se passe. Surtout dans un contexte d'immigration où il y a une discontinuité avec ce qui était avant, souvent de façon brutale. Pour beaucoup, s'il n'y avait pas la religion, je ne sais pas comment ils arriveraient à gérer le chaos dans lequel ils sont [...]. Je pense qu'en intervention c'est quelque chose qu'il faut savoir mobiliser.

Comme dans le cas de Simon, ces répondants proviennent surtout de secteurs particuliers d'intervention (par exemple visites et soutien à domicile, nutrition, DI-TED) où les problèmes vécus par les clients et leur famille semblent exiger d'eux une plus grande sensibilité. Devant une personne en fin de vie ou un parent dont l'enfant souffre d'un handicap sévère, ils introduisent la religion plus spontanément dans leurs démarches afin de leur apporter un soutien, comme l'explique Miranda.

Moi je vous dirais, surtout avec les clients qui sont palliatifs, j'aborde le sujet plus en approfondi, parce que je pense que parfois ça les aide 
[...] Surtout quand je vois que la cliente a des croyances très fortes, moi je l'utilise pour la réconforter et aussi... parce que parfois ça aide. [...] Ça m'arrive d'utiliser la religion pour leur donner une certaine confiance. Pour leur donner une certaine solidité.

Ils ne se limitent pas à poser des questions sur la religion, mais vont jusqu'à évoquer Dieu avec les personnes qu'ils savent croyantes ou les incitent à se tourner vers la religion lors de difficultés. Par exemple, Ariane, qui emploie la religion pour encourager les clients dans des situations de crise, parle de Dieu dans son intervention quand elle juge que c'est propice. Comme les autres intervenants, elle ne réfère à aucune religion en particulier, pour ne pas entrer dans les détails et les différences : "Alors si je mentionne Dieu pour eux, c'est un moment de répit, je le fais. Mais je ne vais pas là où je ne vais pas pouvoir m'en sortir. Je ne vais pas aller discuter de sourates et de Coran et tout cela ». Selon elle, la religion constitue un soutien à ne pas négliger : "C'est vrai que nous sommes dans un lieu laïque, mais si on est là pour aider une clientèle, faisons-le d'une façon qui est... qui leur convient. Si un mot pareil les aide, bien, pourquoi pas? » Elle donne l'exemple d'une cliente des Caraïbes à qui, lors d'une visite à domicile, elle a suggéré de prier afin d'apaiser la douleur provoquée par l'hospitalisation de son fils. Quand la prière occupe une place importante dans la vie des individus, Myriam les encourage aussi à y recourir : "Je regarde avec elle s'il y a des prières qu'elle peut réciter dans telle ou telle situation ou des versets du Coran qu'elle peut lire qui pourraient l'aider dans telle ou telle situation? Parce que je sais que ça fait partie... la prière et la lecture du Coran font partie de ses pratiques ». Comme on l'a vu plus haut, les intervenants n'invitent toutefois pas à la prière des clients n'ayant pas affiché leur appartenance religieuse ou leur foi.

\section{Une dimension plutôt absente}

Selon le témoignage des personnes interviewées dans le cadre de la recherche, la religion ne semble pas représenter un défi de taille dans la pratique, même dans les établissements ancrés dans les secteurs très pluriethniques, principalement en raison de sa relative absence, ce dont témoigne ici Anita, une travailleuse sociale au maintien à domicile : «On n'a pas un défi comme tel parce que ce n'est pas une problématique qui apparaît souvent, beaucoup. Donc, on n'a pas cette préoccupation ». D'une part, la plupart des clients mettent peu cette dimension de l'avant dans la rencontre clinique, comme le souligne Louise : «Les gens sont 
très discrets moi je dirais par rapport à la religion. C'est rare qu'on a des gens qui nous en parlent». D'autre part, aux dires de la majorité des répondants, les requêtes exprimées au nom de la religion sont peu nombreuses, rarement formulées comme un ultimatum et, comme il sera illustré plus loin, il est fréquent que les usagers y renoncent après explications de la part des intervenants. D'ailleurs, presque tous les répondants ont éprouvé des difficultés à répondre à une section du guide d'entretiens où il leur était demandé de documenter un cas clinique problématique associé aux croyances ou pratiques religieuses de leurs clients. Par ailleurs, les intervenants évoluant dans des milieux très pluriethniques classent souvent les immigrants parmi les personnes les plus discrètes en matière de religion, contrairement à l'idée répandue au sein de la société québécoise.

Aux dires des professionnels, les exigences des personnes croyantes ou pratiquantes par rapport aux institutions de santé se situent à un tout autre niveau. Comme l'ensemble de la population québécoise, elles s'attendent d'abord et avant tout à recevoir des services de qualité. Par exemple, elles souhaitent surtout obtenir rapidement un rendezvous et plus de soutien lors de situations difficiles, comme des problèmes de santé ou des difficultés scolaires d'un enfant par exemple. Selon la perception de certains intervenants, en particulier ceux qui jugent que le rôle des institutions de santé en matière de santé doit être limité, la clientèle établit clairement une distinction entre les institutions de santé et les groupes religieux. À ce propos, Ariane, qui travaille surtout auprès d'une clientèle immigrante, déclare: "Nous ne sommes pas un organisme religieux. $\mathrm{Ni}$ une mosquée, ni une synagogue, ni une église... on est le CLSC, l'État».

\section{Différents types de requêtes}

Si la dimension religieuse est relativement absente de la relation de soins, il arrive parfois que des situations particulières se présentent. Elles concernent une grande variété de contraintes religieuses, bien que certaines soient plus récurrentes, comme la question des horaires des rendez-vous par exemple. Plusieurs usagers, dans tous les secteurs d'intervention, préfèrent ne pas consulter lors des jours fériés (par exemple le vendredi chez les musulmans ou le samedi chez les Juifs) ou des célébrations religieuses. Cela dit, de telles requêtes ne sont pas toujours formulées en termes religieux, puisque les clients n'en spécifient 
pas toujours la raison ou avancent parfois d'autres motifs. Éloïse, une psycho-éducatrice au programme DI-TED souligne : « Par exemple, durant la période du jeûne, le ramadan, il y a des clientes qui refusent systématiquement de nous rencontrer. Il y en a d'autres qui vont dire, bien je suis plus fatiguée, est-ce que ça peut attendre et tout ça ». Il est d'ailleurs souvent difficile pour les professionnels de faire la part des véritables demandes liées aux dimensions religieuses, comme lorsque des clients expriment le désir d'être pris en charge par des personnes de même sexe, demande faisant parfois réagir fortement certaines des personnes interviewées. Parmi les autres contraintes religieuses documentées, on note le cas des clients qui souhaitent une nourriture particulière adaptée à leur foi ou encore celui des clients catholiques en perte d'autonomie qui désirent assister à la messe. Certains comportements ou habitudes, attribués à la religion, peuvent aussi provoquer des divergences dans les relations entre les usagers et les professionnels. Des intervenants scolaires parlent de l'opposition de certains parents aux discussions autour de la sexualité à l'école, notamment en raison de leurs croyances religieuses. D'autres, employés au programme famille/enfance, mentionnent l'exemple de mères musulmanes qui observent le jeûne du ramadan pendant leur grossesse ou l'allaitement, bien qu'elles ne soient pas tenues de le faire. Plusieurs donnent aussi l'exemple des maris répondant systématiquement à la place de leur femme, une situation qu'ils attribuent à la religion.

Compte tenu de l'importance de la religion pour de nombreuses personnes au Québec, comment expliquer le faible nombre de demandes en termes religieux dans les CSSS ? La nature des interventions semble en bonne partie responsable, puisque ces interventions diffèrent de celles rencontrées en milieu hospitalier. Il ne s'agit pas d'administrer des médicaments ou de proposer des traitements médicaux. Louise s'exprime à ce propos : «C'est sûr, nous on n'est pas dans les soins médicaux, massifs, comme quand les gens se retrouvent à l'hôpital en situation de crise. On ne gère pas ça à domicile généralement. Fait que c'est sûr qu'on n'a pas à faire de transfusion à domicile ». Les répondants qui interviennent lors de moments chargés de sens, comme la fin de vie ou le handicap par exemple, rencontrent un plus grand nombre de demandes. Ainsi, la religion est absente du travail d'une ergothérapeute, car il est rare qu'une relation de confiance s'instaure avec le client, qu'elle ne rencontre qu'à une ou deux reprises : " Je trouve que nos interventions ne vont pas assez loin pour que ça soit la religion qui 
bloque nos interventions ». Elle établit un lien entre l'intimité et la religion, mais précise que son rôle se limite à proposer des équipements : "C'est sûr qu'on touche aussi des choses qui sont quand même intimes par rapport à l'hygiène des gens, des choses comme ça, fait que des fois, ça, ça peut jouer un peu, mais pas tant que ça, du fait que ce n'est pas nous qui donnons le bain. Nous, on les équipe pour que ça soit fonctionnel». Katia indique pour sa part que la religion apparaît régulièrement dans son travail de nutritionniste, sous forme d'interdits ou de restrictions alimentaires. Seuls quelques intervenants reconnaissent que le faible nombre de demandes ne signifie pas pour autant l'absence de besoins chez les usagers. Par exemple, interrogé sur la question, Jean, un travailleur social œuvrant en milieu scolaire, pointe du doigt l'offre de service : «Les demandes sont forgées en fonction des services qu'on offre. Donc les clients n'ont pas d'attente, s'ils savent qu'il n'y a pas de services. [...] On ne les voit pas, on ne les offre pas, puis on pense que comme on ne les offre pas, ils n'existent pas ». Il ajoute que si les services étaient adaptés, les demandes seraient plus nombreuses.

\section{Respecter le caractère unique de l'individu}

Contrairement aux situations complexes que les professionnels du milieu hospitalier peuvent rencontrer (refus d'une transfusion sanguine ou d'une médication, respect d'un rite funéraire, etc.), les demandes et besoins exprimés par la clientèle des CSSS pour motifs religieux ne semblent pas souvent poser de difficultés particulières pour les professionnels. La plupart déclarent s'ajuster facilement aux attentes des usagers. Ils considèrent qu'il est de leur responsabilité de respecter le caractère unique de l'individu et de le considérer dans sa globalité, d'où l'importance d'offrir des soins adaptés. Aux yeux des répondants, les demandes liées à la religion et les obstacles qu'elle peut générer ne représentent qu'une particularité et une forme de contrainte parmi d'autres. Par exemple, Myriam adapte son intervention aux différents facteurs qui surgissent dans l'intervention : «Oui, comme j'ajuste en fonction d'autres facteurs aussi. L'âge, la personnalité, le niveau de scolarité, la langue, je pense qu'il faut ajuster, [la religion] c'est un facteur parmi d'autres ». Dans le contexte de la prestation de soins et de services, faire la promotion des meilleurs intérêts d'un individu peut signifier avoir à respecter une diversité d'opinions, de croyances, de pratiques et de préférences personnelles. Les répondants tentent donc de proposer 
des solutions respectueuses de la liberté de religion de l'usager, souvent en position de dépendance et de vulnérabilité. Ils estiment aussi que des aménagements mineurs peuvent renforcer la relation thérapeutique. Les exemples abondent: modification de l'horaire des rendez-vous; démarches auprès des autorités religieuses afin qu'un prêtre se déplace à domicile; etc.

La question qui se pose aux intervenants ne consiste donc pas à décider s'ils doivent s'adapter ou pas, mais plutôt jusqu'où et comment ils doivent le faire. Regardons rapidement quelques demandes fondées sur un motif religieux, mentionnées par les intervenants dans le cadre de la recherche et des journées de réflexion organisées par le CSSS de la Montagne, qui font ressortir certaines tensions autour de la religion et qui illustrent bien en même temps quelques-uns des éléments dont les intervenants tiennent compte pour poser des limites aux adaptations. Par exemple, avant de prendre des vitamines prénatales offertes gratuitement par le CLSC, une jeune femme enceinte d'appartenance musulmane consulte une nutritionniste pour s'assurer qu'elles ne contiennent pas de gélatine à base de porc (sa religion lui en interdisant la consommation). Pour l'intervenante, l'information est toutefois difficile à obtenir, car les vitamines proviennent d'une fondation qui envoie de grandes quantités à la fois et elles ne sont donc plus dans leur emballage d'origine. En attente des informations, la nutritionniste s'inquiète de la santé de madame et de son bébé, car elle juge que son alimentation est déficitaire. Une vieille dame copte très malade continue de jeûner, même si son médecin et le prêtre auxquels l'équipe d'intervenants a fait appel pour obtenir des informations tentent de la convaincre qu'elle n'est pas dans l'obligation de le faire en raison de son état de santé et qu'il est impératif qu'elle se nourrisse pour recouvrer la santé. Ces cas cliniques montrent comment les règles de sécurité peuvent servir de balise aux adaptations. De même, les interventions sont également adaptées dans les limites des grands principes de droit, des codes déontologiques, de la considération des dimensions éthiques particulières à chaque situation et selon les ressources disponibles. Les situations suivantes, par exemple, témoignent du souci des intervenants de ne pas adapter les soins au détriment des possibilités de l'offre des soins de santé globale. Un homme juif, qui se rend généralement à la clinique du CSSS pour obtenir ses injections, demande à ce qu'une infirmière vienne lui prodiguer des soins à domicile lors du Sabbat, car il n'est pas en mesure de se déplacer à la clinique cette journée-là (l'usage 
d'un véhicule automobile étant interdit). Les conditions ne le permettant pas (aucune infirmière offrant des soins à domicile n'étant disponible ce jour-là), sa demande est rejetée. De même, un décès est survenu au domicile d'une famille juive chez qui le CSSS avait installé de l'équipement (notamment un lit électrique). Afin de respecter les rites funéraires, la famille exige que les équipements soient retirés le jour même par le CSSS, ce à quoi le fournisseur du Centre n'est pas tenu. Les intervenants éprouvent aussi parfois des difficultés à concilier les valeurs d'intervention ou leurs valeurs personnelles avec les valeurs et pratiques de l'usager. Les questions de l'autonomie et celle de l'égalité hommes/femmes reviennent régulièrement dans les témoignages. Par exemple, une femme maghrébine enceinte demande à être suivie par une femme obstétricienne et, de même, un homme pakistanais veut une femme médecin pour son épouse.

Cela dit, dans la plupart des cas, à travers le dialogue et la négociation, les deux parties en arrivent à des solutions de compromis qui respectent les principales règles des soins de santé et qui tiennent compte des attentes des clients. Par exemple, au bout de deux semaines, l'intervenante met la main sur l'emballage des vitamines distribuées et contacte la dame pour l'informer de l'absence de gélatine à base de porc. La dame copte âgée qui refusait de se nourrir pour des raisons religieuses accepte pour sa part de prendre un supplément liquide. Dans le cas de la famille juive qui demandait que l'équipement soit repris la journée même du décès de leur proche, le fournisseur a accepté de le faire en échange d'un supplément monétaire dont la famille s'est acquittée. L'homme pakistanais qui insistait pour que sa femme soit examinée par une femme médecin a changé d'opinion après que l'intervenant lui ait mentionné, entre autres, que la priorité de l'équipe était le bien-être de son épouse. Quant à la femme musulmane enceinte, elle s'est fait répondre que l'équipe acquiescerait à sa demande si possible, mais qu'elle ne pouvait le lui garantir. En terminant, il est intéressant de mentionner que plusieurs des situations mentionnées par les intervenants peuvent aussi relever d'autres facteurs, comme la méconnaissance des services par les clients ou encore relever du parcours migratoire des clients ou de leurs statuts. 


\section{Conclusion}

Selon les résultats de l'enquête, il semble que la diversité religieuse ne pose pas de défis particuliers dans les CSSS montréalais, en grande partie en raison de la relative absence de demandes faites au nom de la religion par les usagers. Aussi, les intervenants semblent bien s'acquitter de leur mandat professionnel qui consiste à offrir des soins personnalisés et axés sur les besoins des individus. Toutes les personnes interviewées ont mentionné tenir compte des contraintes religieuses des clients lorsqu'ils leur en font part et considèrent aussi la religion comme un levier dans l'intervention, ce qui vient confirmer les résultats des rares études réalisées autour de la question dans d'autres contextes. Par exemple, dans une recherche menée auprès de pédiatres dans une douzaine d'hôpitaux américains, Cadge et Ecklund (2009) ont constaté que tous désiraient respecter le souhait des familles de prier. De même, Cadge et al. (2010) constatent que ces pédiatres considèrent la religion de leurs patients pertinente dans leur pratique clinique quotidienne, tout en hésitant à les interroger directement sur le sujet. Néanmoins, le désir d'adapter les services et de personnaliser les soins observés dans notre recherche ne signifie pas pour autant que la religion soit un sujet neutre. Les valeurs et pratiques des usagers peuvent parfois venir toucher les professionnels dans leur identité individuelle et collective (demande d'adaptation qui brime l'égalité hommes/femmes par exemple). À la suite du projet de recherche, nous avons organisé une journée de réflexion au CSSS de la Montagne sur la place de la religion dans l'intervention. Cette activité, qui rassemblait une soixantaine de professionnels de première ligne, a donné lieu (comme celle organisée en 2008) à des échanges animés et parfois très vifs entre les participants, révélant l'existence de tensions autour de l'aspect privé ou public de la religion. D'ailleurs, tant du côté des professionnels que de celui des usagers, on note un certain malaise à aborder le fait religieux. Les premiers ont peur de choquer ou de heurter les clients, tandis qu'il semble que les seconds ne veuillent pas être jugés à partir de leurs croyances ou pratiques religieuses. Pourquoi les professionnels éprouvent-ils un malaise ? Pourquoi les usagers n'en parlent-ils pas? Notre recherche souligne l'importance de développer de nouvelles recherches pour répondre à ces questions, notamment sur le point de vue des clientèles. Elle soulève aussi des questions sur le rôle du contexte sociopolitique sur l'attitude des professionnels de la santé et de leurs clients. La religion reste un sujet délicat au Québec, ce dont témoignent 
les inquiétudes identitaires ressenties par rapport à la diversification qui se manifestent régulièrement sur la scène publique. Il est fort possible que plusieurs personnes, tant du côté des intervenants que de la clientèle, considèrent que la religion relève de la sphère privée et doive y rester confinée. Dans une étude réalisée au Canada, Reimer-Kirkham (2009) montre à ce propos que les opinions des professionnels de la santé sur l'aspect privé ou public de la religion dans l'espace clinique restent partagées. De plus, la crainte d'être jugés ou étiquetés dans un contexte où la liberté d'expression religieuse dans l'espace public est régulièrement débattue peut aussi empêcher les individus de demander des adaptations pour des raisons religieuses.

Force est de constater que la religion occupe, somme toute, une place assez réduite dans l'intervention de première ligne. Non seulement très peu de professionnels posent directement la question à leurs clients, mais aucun n'aborde cette dimension de façon systématique dans son travail. De façon générale, deux grandes catégories d'intervenants se démarquent : ceux qui se limitent à utiliser la religion uniquement lorsque les usagers la mettent de l'avant et les autres, beaucoup moins nombreux, qui y font appel directement pour apporter un soutien à leurs clients ou développer un lien de confiance. Ces résultats peuvent paraître surprenants à la lumière de tous les travaux qui soulignent une relation positive entre la religion et la santé. Pour les intervenants interviewés, la religion se situe en dehors de leurs compétences professionnelles et ne devient une dimension de l'intervention que si elle occupe une place importante dans la vie des usagers, mais encore faut-il que ceux-ci leur en fassent part. De plus, les demandes d'adaptation liées à la diversité religieuse sont plutôt rares. Un décalage existe donc entre la relative absence de la dimension religieuse dans l'intervention de première ligne et les idées préconçues selon lesquelles les immigrants, en particulier ceux de confession musulmane, exigent des adaptations pour des motifs religieux. Il est vrai que, comparativement au milieu hospitalier, les professionnels de la première ligne se trouvent moins souvent confrontés à des moments chargés de sens pour les clients et leurs familles et davantage propices à la manifestation de la religion. À ce propos, les recherches sur le sujet montrent que la religion devient pertinente lorsque les patients font face à des problèmes de santé sérieux (Eicklund et al. 2007 ; Schneider et Mannel 2006), lorsque les familles doivent prendre des décisions médicales ou lorsqu'un proche est en phase terminale (Cadge et al. 
2009). Il n'est donc pas surprenant de noter que les intervenants qui font appel directement à la religion dans leur travail appartiennent surtout, comme on l'a vu, à certains secteurs d'intervention (visites et soutien à domicile, nutritionniste, DI-TED). Toutefois, contrairement aux résultats d'autres recherches (Cadge et al. 2009 ; Pesut et ReimerKirkham 2010), ces professionnels ne sont pas majoritairement pratiquants ou croyants.

La prise en compte ou non d'éléments religieux dans les soins est une question fort complexe et délicate qui mérite attention, notamment en raison de l'importance de la religion dans la vie d'une proportion croissante de la population québécoise. Il n'existe toutefois pas de réponses définitives. D'un côté, en introduisant la religion dans l'intervention, on court le risque d'essentialiser le vécu des clients, surtout des immigrants, et d'évacuer d'autres facteurs importants de leur expérience, comme le processus migratoire ou la méconnaissance des services par exemple. D'un autre côté, ignorer cette dimension peut venir nuire à l'accessibilité des services sociosanitaires et inciter certaines personnes à se tourner vers d'autres ressources (religieuses ou communautaires par exemple), ce qui ne doit pas être pris à la légère dans un contexte marqué par une sous-utilisation de ces services par les nouveaux arrivants (Le Gall et Cassan 2007). 


\section{Références}

Azdouz, Rachida, 2007, "Les conflits de valeurs et de droits dans le secteur de la santé et des services sociaux », dans Myriam Jézéquel (dir.), Les accommodements raisonnables. Quoi, comment, jusqu'où? Des outils pour tous. Montréal, Éditions Yvon Blais.

Barnes, Linda L. et Susan Sered (dir.), 2005, Religion and Healing in America. New York, Oxford University Press.

Battaglini, Alex, 2005, «Religion, Santé et intervention », dans Solange Lefebvre (dir.), La religion dans la sphère publique : 137-151. Montréal, Les Presses de l'Université de Montréal.

Buryska, James F., 2001, «Assessing the Ethical Weight of Cultural, Religious and Spiritual Claims in the Clinical Context ». Journal of Medical Ethics 27 : 118-122.

Cadge, Wendy et Elaine H. Ecklund, 2007, «Immigration and Religion ». Annual Review of Sociology 33 (1) : 359-379.

__ 2009, «Prayers in the Clinic: How Pediatricians and Pediatric Oncologists Respond». Southern Medical Journal 102 (12) : 12181221.

- et Nicholas Short, 2009, "Religion and Spirituality: A Barrier and a Bridge in the Everyday Professional Work of Pediatric Physicians». Social Problems 56 (4) : 702-721.

Chatters, Linda M., 2000, "Religion and Health: Public Health Research and Practice». Annual Review of Public Health 21:335367.

Cochrane, James R., 2006, «Religion in the Health of Migrant Communities: Asset or Deficit? ». Journal of Ethnic and Migration Studies 32 (4) : 715-736.

Curlin, Farr, John D. Lantos, Chad J. Roach, Sarah A. Sellergren et Marshall H. Chin, 2005, "Religious Characteristics of U.S. Physicians ». Journal of General Internal Medicine 20 : 629-634.

Fadiman, Anne, 1998, The spirit Catches You and You fall Down: A Hmong Child, her American Doctors and the Collision of Two Cultures. New York, Noonday.

Fortin, Sylvie, 2008, «The Paedeatric Clinic as Negociated Social Space ». Anthropology and Medicine 15 (3) : 175-187.

__. Marie-Nathalie Leblanc et Josiane Le Gall, 2008, «Entre la oumma, l'ethnicité et la culture. Le rapport à l'islam chez les musulmans francophones de Montréal ». Diversité urbaine 8 (2): 99-134. 
Hall, David, 1997, Lived Religion in America: Toward a history of Practice. Princeton, NJ., Princeton University Press.

Koenig, Harold G., Michael McCullough et David B. Larson, 2001, The Handbook of Religion and Health. New York, Oxford University Press.

Le Gall, Josiane, 2003, «Le rapport à l'islam des musulmanes shi'ites libanaises ». Anthropologie et Sociétés 27 (1) : 131-148.

- et Christelle Cassan, 2007, "Parcours de soins d'hommes immigrants et découpage sociosanitaire du territoire. Des logiques distinctes ", dans Xavier Leloup et Martha Radice (dir.), Les Nouveaux territoires de l'ethnicité : 57-72. Montréal, Presses de l'Université de Montréal.

LSSS, 2006, Loi sur les services de santé et les services sociaux. L.R.Q. c. S-4.2, art $100: 87$.

Mossière, Géraldine et Deirdre Meintel, 2010, «Tradition and Transition: Immigrant Religious Communities in Urban Contexts (Québec) », dans Richard D. Hecht et Vincent F. Biondo (dir.), Religion in the Practice of Daily Life: 481-508. Santa Barbara, CA, Praeger.

Ong, Aihwa, 1995, «Making the Biopolitical Subject: Cambodian Immigrants, Refugee Medicine and Cultural Citizenship in California ». Social Science and Medecine 40 : 1243-1257.

Orsi, Robert A., 2003, «Is the Study of Lived Religion Irrelevant to the World we Live in? Special Presidential Plenary Address ». Journal for the Scientific Study of Religion 42 (2) : 169-174.

Oxman-Martinez, Jacqueline et Jill Hanley, 2005, «Services médicaux et sociaux pour la population multiculturelle du Canada: les défis de l'équité ». Actes du Forum stratégique Canada 2017, au service de la population multiculturelle du Canada de l'avenir, Patrimoine Canada.

Pesut, Barbara et Sheryl Reimer-Kirkham, 2010, "Situated Clinical Encounters in the Negotiation of Religious and Spiritual Plurality: A Critical Ethnography ». International Journal of Nursing Studies 47 (7) : 815-825.

Reimer-Kirkham, Sheryl, 2009, «Lived Religion: Implication for Nursing Ethics». Nursing Ethics 16 : 406-417.

Schneider, Margaret et Roger Mannel, 2006, «Beacon in the Storm: An Exploration of the Spirituality and Faith of Parents Whose Children Have Cancer ». Issues in Comprehensive Pediatric Nursing $29: 3-24$. 